Beatrice Nickel

\title{
Intertextualität als Intermedialität: Zum Mond fliegen mit Jules Verne, Georges Méliès, Brian Selznick und Martin Scorsese
}

\begin{abstract}
The following paper aims at presenting intermediality as a form of intertextuality by applying Julia Kristeva's extended concept of textuality. Therefore, the literary tradition of the motif of the moon is - to some extent - retraced within the framework of a comparative case study: by analyzing the influence of Jules Verne's moon-fiction Autour de la lune (1869) on Georges Méliès's silent film Le Voyage dans la Lune (1902), which has in turn affected Brian Selznick's best-selling novel The Invention of Hugo Cabret (2007), which has been picturized by Martin Scorsese in his 3D-movie Hugo (2011).
\end{abstract}

Keywords: intermediality; intertextuality; moon fiction; Jules Verne; Georges Méliès; Brian Selznick; Martin Scorsese

\section{Vorbemerkung}

Üblicherweise werden Begriffe wie Zitat, Paraphrase, Intertextualität etc. ausschließlich auf mit sprachlichen Zeichen verfertigte Artefakte angewendet. Erweitert man jedoch den Textbegriff in einem semiotischen Verständnis dahingehend, dass jedes Artefakt aus Zeichen in gewissem Sinne lesbar ist und daher auch Textqualitäten aufweist, so bietet sich die Möglichkeit, das gesamte Spektrum von Intertextualität auch als Semiose aufzufassen, die gattungsübergreifend ist und als Osmose von Texten, Themen und Motiven zwischen den verschiedenen Kunstgattungen beschrieben werden kann. Im Falle des hier vorzustellenden Gegenstandes handelt es sich darum, dass der uralte Menschheitstraum von der Reise zum Mond in Jules Vernes Roman Autour de la Lune (1869) in der sprachlichen Verfassung unter den Bedingungen der industriellen Moderne konkretisiert wurde und zugleich - mit entsprechenden Illustrationen von Émile Bayard und Alphonse de Neuville - auch als visueller Text produziert wurde. ${ }^{1}$ Diese Vorgabe nutzte der Filmemacher Georges Méliès, als er zu Beginn des 20. Jahrhunderts

$1 \mathrm{Zu}$ den zahlreichen literarischen Imaginationen des Fluges zum Mond vgl. Montgomery 1999 und Nicolson 1960.

2 Open Access. (C) 2021 Beatrice Nickel, published by De Gruyter. (c) BY-NC-ND This work is licensed under the Creative Commons Attribution-NonCommercial-NoDerivatives 4.0 International License. https://doi.org/10.1515/9783110642056-037 
einen Kurzfilm zu diesem Thema drehte, der sich explizit auf Jules Vernes Roman bezog (Le Voyage dans la Lune, 1902). Er hatte damit einen Text zweiten Grades erzeugt, in diesem Fall einen kinematographischen Text. Dieser Vorgang wurde rund 100 Jahre später von Brian Selznick zum Prätext seines Romans The Invention of Hugo Cabret (2007). Hier wird das Werk von Georges Méliès in einem ganz umfassenden Sinne durch einen romanesken Metatext vorgeführt, in dessen Zentrum ein anthropomorpher Automat steht, der die zentrale Szene aus Georges Méliès’ Film zeichnen kann. In Selznicks Roman wird durch den Zeichenautomaten das Motiv des Mondfluges als Graphik und als Metatext dritten Grades präsentiert. Wenige Jahre darauf verfilmt Martin Scorsese diesen Roman und schafft damit einen kinematographischen Metatext vierten Grades über Georges Méliès' Bearbeitung von Jules Vernes Roman Autour de la Lune (Hugo, 2011).

\section{Georges Méliès' Le Voyage dans la Lune (UA 1. September 1902)}

Georges Méliès’ 14-minütiger Stummfilm basiert maßgeblich auf Jules Vernes Romanen De la Terre à la Lune (1865) und Autour de la Lune (1869) sowie auf H. G. Wells' Roman The First Men in the Moon (1901). Die nachfolgenden Ausführungen sind auf den Einfluss von Vernes zweitem Mondroman ${ }^{2}$ auf Méliès' Film konzentriert.

Die Handlung des Stummfilms ist schnell zusammengefasst: Auf dem Kongress der Astronomischen Gesellschaft erläutert einer der Wissenschaftler, Professor Barbenfouillis, der von Méliès gespielt wurde und der über die Alliteration mit Barbicane aus Vernes Mondromanen verbunden ist, seinen Plan, mittels einer Kapsel, die aus einer großen Kanone abgeschossen werden soll, zum Mond zu fliegen. Der Zuschauer wird nun zunächst Zeuge der Vorbereitungen, bis der Professor und fünf weitere Astronauten die Kapsel feierlich besteigen und diese danach in den Weltraum abgeschossen wird. Ab diesem Moment bewegt sie sich immer näher auf den Mond zu, und schließlich erscheint auf dem Bildschirm das folgende Szenario: Die Kapsel kollidiert mit dem Mond, und zwar trifft sie in

2 Autour de la lune ist vom 4. November bis zum 8. Dezember 1869 als Fortsetzungsroman im Journal des débats politiques et littéraires erschienen, und im Jahre 1870 wurde er vom berühmten Verleger Pierre-Jules Hetzel in Buchform publiziert. Ich habe diesen und nicht seinen Vorgängerroman mit dem Titel De la terre à la lune (1865) gewählt, weil das thematische Hauptgewicht dort auf den Vorbereitungen des Mondfluges liegt, es mir ja aber gerade um den Mondflug geht. 
das rechte Auge des spätestens seit Plutarchs De facie in orbe lunae bekannten Gesichtes des Mondes bzw. des ,Mannes im Mond“ oder noch genauer der ,Frau im Mond'; denn es handelt sich um das Gesicht der Stummfilmschauspielerin Bleuette Bernon, die in mehreren von Méliès’ Filmen mitgewirkt hat. Auch in Vernes Autour de la Lune erscheint der Mond als eine Frau, und zwar sowohl verbal, nämlich in Michel Ardans Vorstellung („la charmante Astarté, la reine des nuits, la fille de Latone et de Jupiter, la jeune du radieux Apollon!“ (Verne 1977 [1872], 100), als auch visuell. Die folgende in Vernes Roman eingefügte Illustration zeigt die weibliche Gestalt des Mondes, und zwar ohne Gesicht:

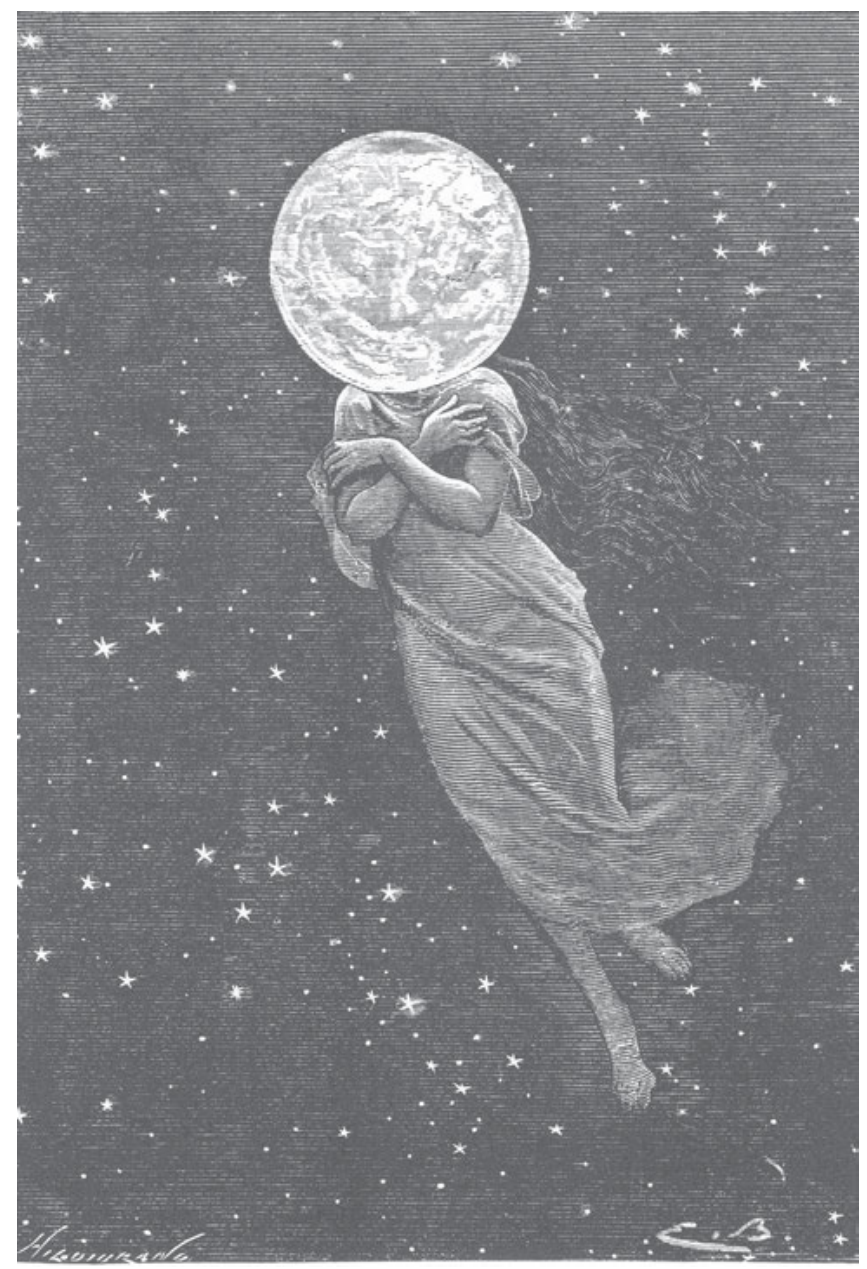

Abb. 1: Illustration zur weiblichen Gestalt des Mondes (Verne 1977 [1872], 101). 
Der Mond erscheint hier konkret in Gestalt der Göttin Selene/Luna, wenn auch unter Aussparung des Gesichtes, das - wie in Méliès' Stummfilm - auch in den nachfolgend untersuchten medialen Transpositionen von großer Bedeutung ist und mit dessen Hilfe jeweils nonverbale Kommunikation betrieben wird. In Méliès' Film wirkt das weibliche Mondgesicht im Moment des Zusammenstoßes sehr traurig, es vergießt sogar eine Träne. Noch besser zu erkennen als im Film ist dies in der entsprechenden, von Méliès gestalteten Vorlage, die bezeichnenderweise den Titel En plein dans l'œil (9 $9^{\mathrm{e}}$ tableau) trägt. ${ }^{3}$

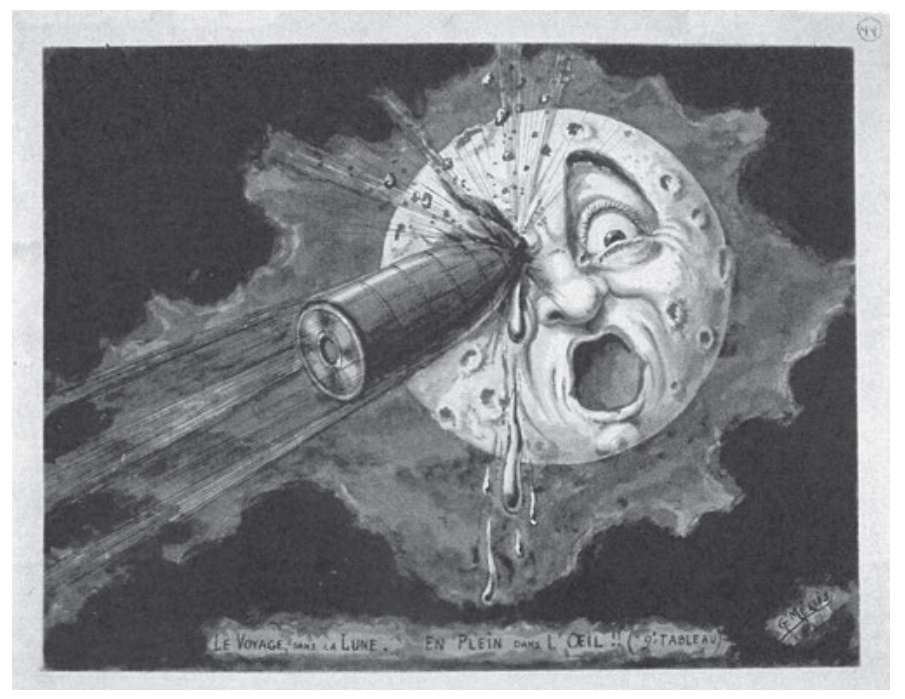

Abb. 2: Georges Méliès, Le Voyage dans la Lune, Screenshot (6m15s)

Méliès führt hier vor, was passiert, wenn die moderne Wissenschaft auf die Natur trifft, die allerdings stark anthropomorphisiert erscheint (vgl. Dagrada 2014, 245-262): „On peut y lire l'avancée du progrès scientifique de l'époque, un contact brutal entre la science et l'état de la nature.“ (Bromberg 2011) Dem Zuschauer wird an dieser Stelle im Film (6m15s) ein close-up des Mondgesichtes präsentiert. Auf dieses close-up des traurigen Mondgesichtes folgt völlig unvermittelt der Blick auf die Mondoberfläche, auf der die Reisenden gelandet sind. (Vgl. Frazer 1979, 96) Wir haben es hier mit der Technik der „,vue à transformations“ (Méliès 2008 [1907], 198) zu tun, die Méliès oft eingesetzt hat. Die Landung

3 Abgedruckt in Malthête/Mannoni 2008, 129. 
auf dem Mond ermöglicht es den Wissenschaftlern, die Kapsel zu verlassen und die ihnen unbekannte Landschaft zu erkunden. Sie treffen hier sowohl auf überdimensional große Pilze als auch auf die Mondbewohner, die Seleniten, die in der Lage sind, plötzlich zu explodieren und unsichtbar zu werden, sobald sie mit einem Regenschirm berührt werden. Die ganze Szenerie auf dem Mond zeichnet sich durch einen explizit anti-illusionistischen, bizarren, märchenhaften Charakter aus. Es handelt sich hierbei um eine Ästhetik, die dadaistische und surrealistische Kompositionen und Bildwelten vorausahnen lässt. Schließlich müssen die Astronauten fliehen, was ihnen auch gelingt, und die Kapsel stürzt - allerdings mit einem Seleniten an Bord - vom Mond in Richtung der Erde ab. Dort landet sie nach ihrem Flug im Meer. Wie in Vernes Roman werden die Astronauten nach ihrer Rückkehr wie Helden gefeiert.

Den Mondreisenden in Méliès' Film wird ein Orden verliehen, und Professor Barbenfouillis, der den entscheidenden Impuls zum Mondflug gegeben hat, wird schließlich eine Statue errichtet, und zwar in einer Pose, die deutlich macht, dass der Wissenschaftler den Mond besiegt hat und ihn mit dem Fuß auf den Erdboden drückt. Diese Szene bildet das Schlussbild, und ihre Inschrift ist für den Zuschauer lesbar: „Labor Omnia Vincit“. Dieses Motto ist dabei Vergils berühmtem Spruch „Omnia vincit Amor““4 nachempfunden. Betrifft dieser ausschließlich den zwischenmenschlichen Bereich, so macht die von Méliès vorgeführte Inschrift deutlich, dass der Mensch durch seine Arbeit und mühevollen Anstrengungen alles - in diesem Fall den Mond als Sinnbild der Natur - besiegen kann, was eher als ironischer Kommentar denn als ernste Aussage aufzufassen ist. (Vgl. Frazer 1979, 98) Eine thematische Nähe zu späteren futuristischen Technikphantasien ist jedoch nicht $\mathrm{zu}$ leugnen. ${ }^{5}$

Auch wenn Jules Vernes zwei Mondfiktionen die maßgeblichen Quellen für Méliès' Verfilmung gewesen sind, so besteht ein wesentlicher Unterschied zur Vorlage vor allem darin, dass es den Protagonisten in Vernes Romanen nicht gelingt, auf dem Mond zu landen, und der Autor aus diesem Grund keine (spekulativen) Angaben über das Leben auf dem Mond zu machen braucht. ${ }^{6}$ Im Gegensatz hierzu handelt der Hauptteil von Méliès' Film von den Lebensformen auf dem Mond (Seleniten, übergroße Pilze etc.). Auch die Intention ist jeweils eine andere. Ging es dem französischen Autor des 19. Jahrhunderts - neben der Unterhaltung seiner Leser - vor allem auch um eine Vulgarisierung naturwissenschaftlicher

4 Der Spruch geht zurück auf ein Zitat aus Vergils 10. Ekloge (10, 69).

5 Vgl. hierzu vor allem Marinettis Manifest Tuons le clair de lune! (1909).

6 Die Frage, ob der Mond bewohnt sei oder nicht, ist zur Zeit Jules Vernes noch völlig offen. Vgl. hierzu Cotardière 2004, 146 und Nickel 2013, 119. 
Erkenntnisse über den Mond, so dient das Mondthema dem Filmemacher vornehmlich dazu, seine Kunst in Szene zu setzen und dem Zuschauer eine imaginäre Traumwelt zu präsentieren. Im Gegensatz zu Vernes Roman erhebt Le Voyage dans la Lune nicht nur „keinerlei Anspruch auf Plausibilität oder Realismus“ (Spiegel 2007, 94), sondern betont gerade seine Fiktionalität. Damit wird „die Tricktechnik Selbstzweck und eigentliches Zentrum des Interesses“ (Spiegel 2007, 94).

Mag auch die Vulgarisierung zeitgenössischer naturwissenschaftlicher Erkenntnisse eines der primären Ziele Vernes gewesen sein, ${ }^{7}$ so darf dies nicht den Blick darauf verstellen, dass schon in Vernes Roman weitere Dimensionen hinzukommen, die Méliès' Umgang mit dem Mondthema vorbereitet haben: Erstens lässt Verne auf dem sehr begrenzten Raum seines imaginierten Projektils zwei Weltanschauungen aufeinandertreffen: Die beiden Mondreisenden Impey Barbicane und der Kapitän Nicholl stehen für die moderne Wissenschaft (Mathematik und Astronomie) und Michel Ardan für die Kunst und die Abenteuerlust. ${ }^{8}$ Diese beiden Blickwinkel unterscheiden sich stark voneinander, zum Beispiel wenn die Romanfiguren die Mondoberfläche betrachten:

Mais tandis que son imagination [scil. l'imagination de Michel Ardan; B.N.] courait ainsi ,les mers', ses graves compagnons considéraient plus géographiquement les choses. Ils apprenaient par cœur ce monde nouveau. Ils en mesuraient les angles et les diamètres. [...] Ce qui, d'ailleurs, était parfaitement indifférent au digne Michel. (Verne 1977 [1872], 110)

Der Autor nutzt die Dualität der möglichen Betrachtungsweisen (wissenschaftlich-objektiv und künstlerisch-subjektiv) dabei vor allem dazu, um „die Partialperspektiven der Romanfiguren ebenso wie die im Roman repräsentierten Wissensformen in ein dialogisches und bisweilen kritisches Verhältnis zueinander“ (Schneider 2010, 100) zu setzen. Insofern Vernes Roman nicht nur wissenschaftliche Fakten ,erzählt', sondern auch poetischen Imaginationen und intuitiven Assoziationen einen breiten Raum bietet, präsentiert er dem Leser einen umfassenden Blick auf den Mond.

Zweitens trägt Verne in seinem Roman auch dem Aspekt Rechnung, dass der Mond - vor allem durch seine relative Nähe zur Erde - seit jeher für den Menschen

7 Wie fundiert die von Jules Verne in seinem Roman vermittelten naturwissenschaftlichen Erkenntnisse sind, geht nicht zuletzt auch daraus hervor, dass die Raumfahrtmission Apollo 11 der NASA tatsächlich vieles von dem eingelöst hat, was Jules Verne schon 100 Jahre früher beschrieben hatte. Vgl. hierzu Unwin 2000, 48, Nickel 2013, 120-121 und http://lexikon.astronomie. info/satelliten/julesverne/index.html. Zu den realen Raumfahrtmissionen vgl. Geiss 2009 und Roussel 2008, 141-143.

8 Repräsentativ sei hier auf das elfte Kapitel in Autour de la Lune hingewiesen: Fantaisie et réalisme. Vgl. hierzu Unwin 2000, 49-50 und Capitanio 2000, 62-64. 
ein Faszinosum darstellt. Zwar dient das Thema des Mondes im Roman zunächst der Vorführung eines wissenschaftlichen Forschungsprojekts, aber dennoch wird der Mond auch als ein Objekt der menschlichen Faszination dargestellt. Dies gilt dabei ausdrücklich nicht nur für den Künstler Michel Ardan, sondern auch für die beiden Forscher an Bord des Projektils, die vom Mond wie verzaubert sind. ${ }^{9}$ Damit verbindet Verne im Mondthema Naturwissenschaft und Irrationales miteinander: „Diese Faszination steht der prinzipiellen Tendenz zur ,Entzauberung“ des Mondes, wie sie die Geschichte poetisch fiktionalisierter Mondflüge aufweist, entgegen.“ (Nickel 2013, 120) Deutlich stärker als Vernes Roman hat Méliès’ Stummfilm dieser ,Entzauberung، entgegengewirkt.

Das Thema des Mondes gibt Méliès zunächst und vor allem die Möglichkeit, seinem Anliegen nach einer explizit nicht-mimetischen Filmkunst Ausdruck zu verleihen. Denn - ganz anders als zu derselben Zeit die Brüder Lumière ${ }^{10}$ - wollte Méliès gerade nicht mittels Realitätseffekten die Illusion von Authentizität und Wirklichkeitsnähe erzeugen, sondern vielmehr filmische Traumwelten durch „Wunderbarkeitseffekte“ (Spiegel 2007, 92) schaffen: ${ }^{11}$ „Mit der zunehmenden Inszenierung eigener Erzählwelten öffnet er das Medium Film für die Darstellung wunderbarer Welten. “ (Hartmann 2015; vgl. hierzu auch Spiegel 2007, 29-41) Den Stummfilm als einen der ersten Repräsentanten der filmischen Science-Fiction zu bezeichnen, muss aufgrund seines explizit anti-illusionistischen Charakters als unzutreffend entlarvt werden. (Vgl. Spiegel 2007, 94-95)

Le Voyage dans la Lune lässt sich mit Fug und Recht als filmisches Manifest bzw. filmisch realisierte Programmatik emblematischer Bedeutung für Méliès' Werk auffassen. Gerade die Sequenz, in der die Kapsel mitsamt den sechs Astronauten im rechten Auge des Mondes landet, gibt einen exemplarischen Einblick in Méliès’ technische Fähigkeiten. Insofern ist es wenig verwunderlich, dass eben diese Szene eine bedeutende Rolle in Selznicks Roman spielen wird.

\footnotetext{
9 Vgl. hierzu beispielsweise das folgende Zitat aus Autour de la lune: „Quel ravissement de jeter un regard sur ce monde que l'œil humain n’a jamais entrevu !“ (Verne 1977 [1872], 140)

$10 \mathrm{Zu}$ den Filmen der Brüder Lumière vgl. beispielsweise Sadoul 1949, 18-26.

11 Dies gilt nicht für Méliès' erste Filme, die noch stark an den Werken der Brüder Lumière orientiert sind: „Les premiers films de Méliès n’ont aucune originalité. [...] L’originalité de Méliès se révèle quand il aborde le truquage et consacre quatre-vingt mille francs-or à construire, en 1897, un studio dans sa belle propriété de Montreuil, aux portes de Paris.“ (Sadoul 1949, 27)
} 


\section{Brian Selznick, The Invention of Hugo Cabret (2007)}

Mit dem Erfolg von Apollo 11 ist die Utopie von der Mondreise eingelöst, und es beginnt die Phase einer metafiktionalen Reflexion über die mediale Verarbeitung der Utopie von der Mondreise. Bemerkenswert ist hier der verbal-visuelle Roman ${ }^{12}$ The Invention of Hugo Cabret (2007) von Brian Selznick. Hier geht es um die Rekonstruktion eines Zeichenautomaten, der die Filmszenarien von Georges Méliès zeichnen kann - darunter auch die Bilder aus Méliès' Stummfilm Le Voyage dans la Lune (1902). Zu denken ist hier an einen Automaten wie denjenigen, den der Schweizer Henri Maillardet bereits zu Beginn des 19. Jahrhunderts konstruiert hatte und der vier Bilder zeichnen und drei Gedichte aufschreiben konnte.

Nachdem der Automat repariert ist, ist er imstande, jenes Bild aus Méliès' Film Le Voyage dans la Lune zu zeichnen, das zeigt, wie die Raumkapsel mit dem rechten Auge des Mondes zusammenstößt. (Vgl. Selznick 2007, 252-253) Es handelt sich dabei um das zentrale Bild aus Méliès' Stummfilm. Als zentral ist dieses Bild vor allem deshalb zu bezeichnen, weil das weinende Mondgesicht auf paradigmatische Weise Méliès' anti-illusionistische Filmkonzeption repräsentiert. (Vgl. Spiegel 2007, 94) ${ }^{13}$ Bei Le voyage dans la Lune soll es sich übrigens um den Lieblingsfilm von Hugos verstorbenem Vater handeln (vgl. Selznick 2007, 354). Diese intermediale Referenz auf den französischen Filmemacher wird in Selznicks Roman insofern offengelegt, als der Automat seine Arbeit nicht beendet, nachdem er dieses Bild gezeichnet hat, sondern folgendermaßen fortfährt:

That's when the children [scil. Hugo and Isabelle; B.N.] realized that the mechanical man wasn't finished. It seemed to have stopped mid-line, as if it was pausing. Hugo watched as one more time the mechanical man dipped its pen into the ink. Then it moved its hand into position and... signed a name. (Selznick 2007, 259)

Durch die Aposiopese verlängert Selznick gekonnt den Spannungsbogen und steigert dadurch die Aufmerksamkeit des Lesers. Zugleich hebt er die wichtige

12 Der Autor selbst hat als Untertitel folgende Gattungsbezeichnung gewählt, um die prinzipielle Gleichwertigkeit der schriftlichen und der bildlichen Anteile deutlich zu machen: „A Novel in Words and Pictures“. Auch John Schwartz hat in seiner Rezension darauf hingewiesen, dass Selznicks Roman nicht als Graphic Novel zu klassifizieren sei: „This is much more than a graphic novel: it is more like a silent film on paper“. (Schwarz 2007)

13 Es ist daher wenig verwunderlich, dass mehrere Monographien zu Méliès und seinen Filmen gerade dieses Bild als Cover verwenden. Vgl. hierzu beispielsweise Gaudreault 2008 und Ezra 2000. 
Bedeutung der Person, deren Name nachfolgend als Unterschrift erscheint, hervor: Georges Méliès. Mit dieser Unterschrift beginnt der zweite Teil des Romans: Standen im ersten Teil die Versuche Hugos, den Zeichenautomaten, den sein toter Vater einst erfunden hat, zu rekonstruieren, um eine vermeintliche Botschaft von diesem zu empfangen, im Fokus, so liegt das Hauptaugenmerk von diesem Moment an auf Méliès, der den Zeichenautomaten gebaut haben soll. Der Automat fungiert daher als Bindeglied zwischen den beiden Teilen des Romans:

\begin{abstract}
These two stories intersect most powerfully at the figure of the automaton, which appears at first like the attraction it was built to be. Yet over the course of the movie, the automaton functions as a narrative device that propels the plot and that unites the film's dual interests of the written word and the moving image into a single focal point that closely resembles early cinema itself, with its own reliance on writing to supplement and complement its images. (Clement und Long 2012)
\end{abstract}

Was als tragische Geschichte um die unsichere Zukunft eines Waisenkindes begonnen hat, wird im zweiten Teil primär zu einer Hommage an das frühe Kino und an Méliès. In diesem zweiten Teil begibt Hugo sich auf die Spuren des weltbekannten französischen Filmemachers. In einem fiktiven Buch, das den Titel The Invention of Dreams: The Story of the First Movies Ever Made (1930) trägt, ${ }^{14}$ findet Hugo folgenden Eintrag über Méliès:

The filmmaker Georges Méliès began his career as a magician and he owned a theatre of magic in Paris. This connection with magic helped him immediately understand what the new medium of film was capable of. He was among the first to demonstrate that film didn't have to reflect real life. He quickly realized that film had the power to capture dreams. Méliès is widely credited with perfecting the substitution trick, which made it possible for things to appear and disappear on screen, as if by magic. This changed the face of movies forever. (Selznick 2007, 354)

Auf diesen Eintrag folgen zwei Originalfotos von Méliès (vgl. Selznick 2007, 356359), mittels derer auf den Beginn seiner Karriere als Zauberer verwiesen wird. Im Fokus des Romans steht jedoch seine Tätigkeit als Filmemacher. Denn nachdem Hugo und seine Freundin Isabelle Originalzeichnungen aus Méliès' Filmen versteckt in einem Geheimfach in einem Kleiderschrank gefunden und den Filmpionier mit ihnen konfrontiert haben, wird ihnen klar, dass Méliès mit seiner Vergangenheit abgeschlossen und sich damit abgefunden hat, in Vergessenheit geraten zu sein. Daraufhin sorgen die beiden Kinder dafür, dass Méliès Film Le

14 Handelt es sich auch um einen rein fiktiven Titel eines ebenso fiktiven Buches, so ist die Parallele zum Titel von Selznicks Roman offensichtlich und sehr aussagekräftig. 
voyage dans la lune öffentlich gezeigt und dem Filmemacher schließlich zu der ihm gebührenden Anerkennung verholfen wird.

Der Schluss des Romans ist als uneingeschränktes Happy Ending gestaltet, und zwar sowohl für Hugo als auch für Méliès. Denn der Filmpionier erfährt eine Ehrung von der französischen Filmakademie, und Méliès nimmt die Vollwaise Hugo bei sich auf.

Im Fall von Selznicks Roman haben wir es mit Blick auf Méliès’ Stummfilm erneut mit einem Medienwechsel zu tun, und zwar mit einem solchen, der auf Vernes Mondfiktion verweist, die ja ebenfalls die Medien Schrift und Bild nutzt, auch wenn hier - anders als in Selznicks Roman - noch nicht von der prinzipiellen Gleichwertigkeit beider Zeichensysteme gesprochen werden kann, zumal die Abbildungen in Vernes Romanen ausschließlich illustrierenden Charakters sind und damit den Text des Romans lediglich in ein anderes Medium transponieren, statt die Handlung voranzutreiben, wie dies in Selznicks Roman der Fall ist. Nichtsdestoweniger präsentiert Selznick das Motiv des Mondfluges mit Blick auf Vernes Vorlage als Metatext dritten Grades.

\section{Ausblick: Martin Scorsese, Hugo (2011)}

Martin Scorseses erster 3D-Film, der für elf Oscars nominiert und mit fünf Oscars ausgezeichnet wurde, basiert maßgeblich auf Brian Selznicks Roman The Invention of Hugo Cabret, dem - vor allem durch die zahlreichen Bilder - selbst immer wieder kinematographische Qualitäten zugeschrieben wurden. ${ }^{15}$ Auch Scorseses Werk feiert den Filmpionier Méliès (gespielt von Ben Kingsley), und zwar in Méliès' ureigenem Medium, dem Film. Auch bei Scorsese schreibt der Zeichenautomat den Namen des berühmten französischen Filmemachers unter das Bild des weinenden Mondgesichtes.

Wie Selznicks Roman stellt auch Scorseses Film eine Hommage an das frühe Kino dar: In einer Rezension auf Spiegel Online wurde Hugo als „traumhafte Liebeserklärung an die Magie des Kinos“ (Borcholte 2012) bezeichnet. Hat Méliès dem Zuschauer in seinen Filmen Traumwelten vor Augen geführt, so gilt dies uneingeschränkt auch für Scorsese und Hugo:

15 Vgl. hierzu beispielsweise Clement und Long 2012: „The novel seems almost cinematic, an impression reinforced by the number of stills used in the book to help bring the reader into the characters' experiences of movie watching. Transferring such a novel to screen seems an obvious move, the fulfillment perhaps of the novel's own desire to transcend its medium, and a kind of acknowledgement of the novel's integration of film into the novel's own form and story.“ 
Dass Scorsese seine Verbeugung vor dem Erfindungsreichtum der Kinoväter ausgerechnet in 3D drehte, sollte nur auf den ersten Blick irritieren: Wie könnte man sich treffender vor der Phantasie eines Georges Méliès verneigen als mit Hilfe der modernsten Illusionstechnik, die Hollywood zu bieten hat? Hugo Cabret ist ein Wunderwerk, das alle Register der Maschinerie zieht, die Kino zu einem der staunenswertesten Kulturmedien gemacht haben: Kaum etwas an diesem 170 Millionen teuren Film ist echt, die meisten Kulissen sind digital oder wurden liebevoll gezeichnet. (Borcholte 2012)

\section{Schlussbetrachtung}

Mit Blick auf Jules Vernes Roman Autour de la Lune wurden Georges Méliès' Stummfilm Le voyage dans la Lune, Brian Selznicks verbal-visueller Roman The Invention of Hugo Cabret und schließlich Martin Scorseses 3D-Film Hugo als Fälle verschiedener Grade von Intertextualität im weiteren Sinne und Intermedialität vorgestellt. Die einzelnen Grade der Intertextualität weisen dabei unterschiedlich gestaltete Verhältnisse zum Ausgangstext auf. Im Falle von Méliès' Film ist Intertextualität untrennbar mit einem Medienwechsel vom Buch zum Film verbunden. Selznicks novel in words and pictures kehrt zwar zum Ausgangsmedium, dem Buch, zurück, ist aber stärker auf Méliès’ Bearbeitung von Vernes Roman bezogen als auf diesen Roman selbst. Insofern hier explizit auf das Medium Film im Allgemeinen ${ }^{16}$ und Méliès' Stummfilme im Besonderen - und vor allem auf Le voyage dans la Lune - verwiesen wird, haben wir es mit einer remediation $\mathrm{zu}$ tun. ${ }^{17}$ Scorseses Film stellt mit Blick auf Selznicks Roman einen Medienwechsel, mit Blick auf Méliès' Bearbeitung von Vernes Roman jedoch eine Rückkehr zum Medium der Vorlage dar. Es handelt sich hierbei um einen kinematographischen Metatext vierten Grades über Vernes Roman, auch wenn diese Vorlage nur indirekt, in Form der Bearbeitung von Méliès und seiner Vermittlung, greifbar wird.

16 Beispielsweise enthält der Roman mehrere Originalzeichnungen von Méliès für Filmszenen (vgl. Selznick 2007, 284-297) und das Standbild des in einen Bahnhof einfahrenden Zuges aus dem Film L'Arrivée d'un train en gare de La Ciotat (1895) der Brüder Lumière (Selznick 2007, 348349).

17 Zum Konzept der remediation vgl. Bolter und Grusin 2000. 


\section{Literaturverzeichnis}

Bolter, Jay David, und Richard Grusin. Remediation. Understanding New Media. Cambridge und London: MIT Press, 2000.

Borcholte, Andreas. „3-D-Märchen Hugo Cabret: Kleiner Held, großer Bahnhof“. Spiegel Online, 07. Februar 2012. http://www.spiegel.de/kultur/kino/3-d-maerchen-hugo-cabret-kleinerheld-grosser-bahnhof-a-813650.html (14. Februar 2018).

Bromberg, Serge. Le voyage dans la Lune de Georges Méliès était l'Avatar de l'époque. http:// www.vousnousils.fr/2011/12/09/serge-bromberg-le-voyage-dans-la-lune-de-georgesmelies-etait-l-avatar-de-l-epoque-517962 (15. Februar 2018).

Capitanio, Sarah. ,,L'Ici-bas' and ,l'Au-delà'... but Not as they Knew it. Realism, Utopianism and Science Fiction in the Novels of Jules Verne“. Jules Verne: Narratives of Modernity. Hg. Edmund J. Smyth. Liverpool: University Press, 2000. 60-77.

Clement, Jennifer, und Christian B. Long. „Hugo, Remediation, and the Cinema of Attractions, or, The Adaptation of Hugo Cabret“. Senses of Cinema 63 (July 2012). http:// sensesofcinema.com/2012/feature-articles/hugo-remediation-and-the-cinema-ofattractions-or-the-adaptation-of-hugo-cabret/ (14. Februar 2018).

Cotardière, Philippe de la (Hg.). Jules Verne. De la science à l'imaginaire. Paris: Larousse, 2004. Dagrada, Elena. „Entre la Terre et la Lune, ou de l'anthropomorphisation des astres“. Méliès, carrefour des attractions. Hg. André Gaudreault und Laurent Le Forestier. Rennes: Presses Universitaires, 2014. 245-262.

Ezra, Elizabeth. Georges Méliès. The Birth of the Auteur. Manchester und New York: Manchester University Press, 2000.

Frazer, John. Artificially Arranged Scenes. The Films of Georges Méliès. Boston: G. K. Hall \& Co., 1979.

Gaudreault, André. Cinéma et attraction. Pour une nouvelle histoire de cinématographe. Paris: CNRS Éditions, 2008.

Geiss, Johannes. „Die Apollo Mondlandungen“. Science \& Fiction. Imaginationen und Realität des Weltraums. Hg. Thomas Myrach, Tristan Weddigen, Jasmine Wohlwend und Margarita Zwahlen. Bern, Stuttgart und Wien: Haupt, 2009. 16-50.

Hartmann, Jörg. „Träumen Astronomen vom Mann im Mond? Keplers Somnium (1634) und Méliès' La lune à un mètre (1898) im geopoetischen Vergleich“. reflex 6 (2015). https://publikationen.uni-tuebingen.de/xmlui/bitstream/handle/10900/69382/ Hartmann\%20final.pdf?sequence=1\&isAllowed=y] (15. Februar 2018).

http://lexikon.astronomie.info/satelliten/julesverne/index.html (15. Februar 2018)

Hugo. Reg. Martin Scorsese. Paramount, 2011.

Malthête, Jacques, und Laurent Mannoni. L'œuvre de Georges Méliès. Paris: Éditions de La Martinière, 2008.

Marinetti, Filippo Tommaso. „Tuons le clair de lune!“ Poesia 5 (1909): 1-9.

Méliès, Georges. „Les vues cinématographiques (1907)“. Cinéma et attraction. Pour une nouvelle histoire de cinématographe. Hg. André Gaudreault. Paris: CNRS Éditions, 2008. 195-222.

Montgomery, Scott L. The Moon \& the Western Imagination. Tucson: University of Arizona Press 1999.

Nickel, Beatrice. „Reisen zum Mond zwischen Wissenschaft und Fiktion“. Komparatistik. Jahrbuch der Deutschen Gesellschaft für Allgemeine und Vergleichende Literaturwissenschaft 2013: 105-124. 
Nicolson, Marjorie Hope. Voyages to the Moon. New York: Macmillian 1960.

Roussel, Gilles. „Un rêve presque aussi vieux que le monde“. Jules Verne - Les mondes inventés. Hg. Agnès Marcetteau. Paris: Somogy éditions, 2008. 136-143.

Sadoul, Georges. Histoire de cinéma mondial. Des origines à nos jours. 9ème édition. Paris: Flammarion, 1949.

Schneider, Steffen. „Enzyklopädie, wissenschaftliche Beobachtung, Beschreibung: Drei Formen von Objektivität und ihre ironische Vermittlung in Jules Vernes Romanen De la Terre à la Lune und Autour de la Lune“. Objektivität und literarische Objektivierung seit 1750. Hg. Niklas Bender und Steffen Schneider. Tübingen: Narr, 2010 (editions lendemains; 22). 99-120.

Schwarz, John. „The Invention of Hugo Cabret“. The New York Times, March 11, 2007. http:// www.nytimes.com/2007/03/11/books/review/Schwartz.t.html?_r=0 (15. Februar 2018).

Selznick, Brian. The Invention of Hugo Cabret. A Novel in Words and Pictures. New York: Scholastic Books, 2007.

Spiegel, Simon. Die Konstitution des Wunderbaren. Zu einer Poetik des Science Fiction Films. Marburg: Schüren, 2007.

Unwin, Timothy. „The Fiction of Science, or the Science of Fiction“. Jules Verne: Narratives of Modernity. Hg. Edmund J. Smyth. Liverpool: University Press, 2000. 46-59.

Verne, Jules. Voyages extraordinaires, Bd. 1,3: Autour de la lune suivi de Un drame dans les airs. Neudruck der Ausgabe Paris 1872. Paris: Bibliothèque d’Éducation et de Récréation J. Hetzel et Cie, 1977.

Beatrice Nickel, PD Dr., ist Akademische Oberrätin am Institut für Allgemeine und Vergleichende Literaturwissenschaft der Ruhr-Universität Bochum. Forschungsschwerpunkte: die Literatur der Frühen Neuzeit, des 18. und 20. Jahrhunderts, Intermedialität, Rezeption, Globalität der Literatur, Literatur und Raum. Publikationen (Auswahl): Texte inmitten der Künste - Intermedialität in romanischen, englischen und deutschen Gedichten nach 1945 (2015), als (Mit-)Herausgeberin: Digital Humanities. Perspektiven der Praxis (2018), Kultur - Landschaft Raum: Dynamiken literarischer Inszenierungen von Kulturlandschaften (2018). 
